\title{
Global nonexistence and blow-up results for a quasi-linear evolution equation with variable-exponent nonlinearities
}

Abita Rahmoune and Benyattou Benabderrahmane

\begin{abstract}
In this paper, we consider a class of quasi-linear parabolic equations with variable exponents,

$$
a(x, t) u_{t}-\Delta_{m(.)} u=f_{p(.)}(u)
$$

in which $f_{p(.)}(u)$ the source term, $a(x, t)>0$ is a nonnegative function, and the exponents of nonlinearity $m(x), p(x)$ are given measurable functions. Under suitable conditions on the given data, a finite-time blow-up result of the solution is shown if the initial datum possesses suitable positive energy, and in this case, we precise estimate for the lifespan $T^{*}$ of the solution. A blow-up of the solution with negative initial energy is also established.
\end{abstract}

Mathematics Subject Classification (2010): 35K92, 35B44, 35A01.

Keywords: Global nonexistence, quasi-linear evolution equation, Sobolev spaces with variable exponents, variable nonlinearity.

\section{Introduction}

Let $\Omega$ be a bounded domain in $\mathbb{R}^{n}, n \geq 1$ with a smooth boundary $\Gamma=\partial \Omega$. We consider the following initial-boundary value problem:

$$
\left\{\begin{array}{c}
a(x, t) u_{t}-\Delta_{m(.)} u=f_{p(.)}(u), \quad x \in \Omega, t>0 \\
u(x, t)=0 \text { on } \Gamma, t \geq 0 \\
u(x, 0)=u_{0}(x), x \in \Omega,
\end{array}\right.
$$

where

$$
\Delta_{m(.)} u=\operatorname{div}\left(|\nabla u|^{m(x)-2} \nabla u\right)
$$


called the $m($.$) -Laplacian operator. This operator can be extended to a monotone$ operator between the space $W_{0}^{1, m(.)}(\Omega)$ and its dual as

$$
\left\{\begin{array}{c}
-\Delta_{m(.)} u: W_{0}^{1, m(.)}(\Omega) \rightarrow W^{-1, m^{\prime}(.)}(\Omega), \\
<-\Delta_{m(.)} u, \phi(x)>_{m(.)}=\int_{\Omega}|\nabla u|^{m(x)-2} \nabla u \nabla \phi(x) \mathrm{d} x, \\
\text { where } 2<m_{1} \leq m(x) \leq m_{2}<\infty .
\end{array}\right.
$$

where $<., .>_{m(.)}$ denotes the duality pairing between $W_{0}^{1, m(.)}(\Omega)$ and $W^{-1, m^{\prime}(.)}(\Omega)$,

$$
\frac{1}{m(x)}+\frac{1}{m^{\prime}(x)}=1
$$

$f_{p(.)}(u)$ is a general source term depends on $p($.$) , the coefficients a(x,$.$) is a nonnega-$ tive function, the exponents $p($.$) and m($.$) are given measurable functions on \bar{\Omega}$ such that:

$$
2<m_{1} \leq m(x) \leq m_{2}<p_{1} \leq p(x) \leq p_{2} \leq m_{*}(x),
$$

where, for any function $\psi$, we set

$$
\psi_{2}=e s s \sup _{x \in \Omega} \psi(x), \quad \psi_{1}=e s s \inf _{x \in \Omega} \psi(x) .
$$

and

$$
m_{*}(x)=\left\{\begin{array}{l}
\frac{n m(x)}{(n-m(x))_{2}} \text { if } n>m_{2} \\
+\infty \text { if } n \leq m_{2} .
\end{array}\right.
$$

We also assume that $m($.$) satisfies the following Zhikov-Fan uniform local continuity$ condition:

$$
|m(x)-m(y)| \leq \frac{M}{|\log | x-y||}, \text { for all } x, y \text { in } \Omega \text { with }|x-y|<\frac{1}{2}, M>0 .
$$

A considerable effort has been devoted to the study of problem (1.1) in the case of constant variable when $p(x)=p=$ constant and $m(x)=m=$ constant. The problem (1.1) with the usual $m$-Laplacian operator $\Delta_{m} u=\operatorname{div}\left(|\nabla u|^{m-2} \nabla u\right),(m=$ constant $\geq 2) ;\left(m=2, \Delta_{m} u=\Delta u\right)$, has been extensively studied concerning existence, nonexistence and long-time dynamics. For results of the nature and in the case when $p(x)=p=$ constant $\geq 2$ and $m(x)=m=$ constant $>2$, we refer the reader to $[14,18,21]$ related to the equation

$$
a(x) u_{t}-\operatorname{div}\left(|\nabla u|^{m-2} \nabla u\right)=f_{p}(u), x \in \Omega, t>0 .
$$

When $m(x)=m=2, a(x, t)=1$ and $f_{p(.)}(u)=u^{p(x)}$, problem (1.1) becomes the following

$$
u_{t}-\Delta u=u^{p(x)}, x \in \Omega, t>0 .
$$

The problem (1.4) arises from many important mathematical models in engineering and physical sciences. For example, nuclear science, chemical reactions, heat transfer, population dynamics, biological sciences, etc., and have interested a great deal of attention in the research, see $[1,2,4,7,12]$ and the references therein. For problem (1.4), Hua Wang et al. [15] established a blow-up result with positive initial energy under some suitable assumptions on the parameters $p($.$) and u_{0}$. In [12], the authors 
proved that there are non-negative solutions with a blow-up in finite time if and only if $p_{2}>1$. The authors in [20] obtained the solution of problem (1.1) blows up in a finite time when the initial energy is positive. In [8], authors based on the idea as in [5] derived the lower bounds for the time of blow-up if the solutions blow up.

This work is extend the results established in bounded domains to general problem as in (1.1) in the case, when the exponents $m($.$) and p($.$) are given measur-$ able functions on $\bar{\Omega}$ and satisfy $(1.2)$ and $f_{p(.)}(u)$ is a more generalized source term. We note that the presence of the variable-exponent nonlinearities and the coefficient $a(x, t)$ in this problem make analysis in the paper somewhat harder than that in the related ones. The goal of the current project is to study the blow-up phenomenon of solutions to the problem (1.1) in the framework of the Lebesgue and Sobolev spaces with variable exponents, we will establish a blow-up result and give a precise estimate for the lifespan $T^{*}$ of the solution in this case. The method used here is the concavity method. However, because of the presence of the variable-exponent nonlinearities in our problem, our argument is considerably different and it is more abbreviated. The present report is organized as follows. In Sections 2, the Orlicz-Sobolev function spaces are introduced, and a brief description of their main properties are presented. In Sections 3, the blow up for positive initial energy of problem (1.1) is stated. Section 4 provides proof of the blow-up for negative initial energy of problem (1.1).

\section{Preliminaries}

In this section, some well-known results and facts from the theory of Sobolev spaces with variable exponents are recalled and listed (for details, see $[9,10,11,13$, 17]). Throughout the rest of this report, $\Omega$ is assumed to be a bounded domain of $\mathbb{R}^{n}$, $n \geq 2$ with a smooth boundary $\Gamma$, assuming that $p($.$) is a measurable function on \bar{\Omega}$ and satisfy the following Zhikov-Fan uniform local continuity condition:

$$
|p(x)-p(y)| \leq \frac{M}{|\log | x-y||}, \text { for all } x, y \text { in } \Omega \text { with }|x-y|<\frac{1}{2}, M>0 .
$$

Let $p: \Omega \rightarrow[1, \infty]$ be a measurable function. $L^{p(\cdot)}(\Omega)$ denotes the set of measurable functions $u$ on $\Omega$ such that

$$
\varrho_{p(.)}(u)=\int_{\Omega}|u(x)|^{p(x)} \mathrm{d} x .
$$

The variable-exponent space $L^{p(.)}$ equipped with the Luxemburg norm

$$
\|u\|_{p(.)}=\|u\|_{L^{p(.)}(\Omega)}=\inf \left\{\lambda>0, \quad \varrho_{p(.)}\left(\frac{u}{\lambda}\right) \leq 1\right\}
$$

is a Banach space. In general, variable-exponent Lebesgue spaces are similar to classical Lebesgue spaces in many aspects; see the first discussion of $L^{p(x)}(\Omega)$ and $W^{k, p(x)}(\Omega)$ spaces by Kovàcik and Rákosnik in [17].

Here are some properties of the space $L^{p(.)}(\Omega)$, which will be used in the study of a problem (1.1). 
- It follows directly from the definition of the norm that

$$
\min \left(\|u\|_{p(.)}^{p_{1}},\|u\|_{p(.)}^{p_{2}}\right) \leq \varrho_{p(.)}(u) \leq \max \left(\|u\|_{p(.)}^{p_{1}},\|u\|_{p(.)}^{p_{2}}\right) .
$$

- The following generalized Hölder inequality

$$
\int_{\Omega}|u(x) v(x)| \mathrm{d} x \leq\left(\frac{1}{p_{1}}+\frac{1}{\left(p_{1}\right)^{\prime}}\right)\|u\|_{p(x)}\|v\|_{p^{\prime}(x)} \leq 2\|u\|_{p(x)}\|v\|_{p^{\prime}(x)}
$$

applies for all $u \in L^{p(.)}(\Omega), v \in L^{p^{\prime}(.)}(\Omega)$ with $p(x) \in(1, \infty), p^{\prime}(x)=\frac{p(x)}{p(x)-1}$.

- If condition (2.4) is fulfilled, $\Omega$ has a finite measure, and $p, q$ are variable exponents such that $p(x) \leq q(x)$ almost everywhere in $\Omega$, then the embedding $L^{q(\cdot)}(\Omega) \hookrightarrow L^{p(\cdot)}(\Omega)$ is continuous.

- The Sobolev space $W_{0}^{1, p(.)}(\Omega)$ with $p(x) \in\left[p_{1}, p_{2}\right] \subset(1, \infty)$, and $\frac{1}{p(x)}+\frac{1}{p^{\prime}(x)}=1$, is defined as

$$
\left\{\begin{array}{c}
W_{0}^{1, p(.)}(\Omega)=\left\{\left.u \in L^{p(.)}(\Omega)|| \nabla u\right|^{p(x)} \in L^{1}(\Omega), u=0 \text { on } \partial \Omega\right\}, \\
\|u\|_{W_{0}^{1, p(.)}(\Omega)}=\|u\|_{1, p(.)}=\sum_{i}\left\|D_{i} u\right\|_{p(.), \Omega}+\|u\|_{p(.), \Omega},
\end{array}\right\}
$$

and $W^{-1, p^{\prime}(.)}(\Omega)$ is defined in the same way as the usual Sobolev spaces (see $[9])$.

- An equivalent norm of $W_{0}^{1, p(.)}(\Omega)$ is given by

$$
\|u\|_{W_{0}^{1, p(.)}(\Omega)}=\|\nabla u\|_{p(.), \Omega} \cdot
$$

Furthermore, we set $W_{0}^{1, p(.)}(\Omega)$, to be the closure of $C_{0}^{\infty}(\Omega)$ in $W^{1, p(.)}(\Omega)$. Here we note that the space $W_{0}^{1, p(.)}(\Omega)$ is usually defined in a different way for the variable exponent case. However (see Diening et al [9]), both definitions are equivalent under (1.3). The $\left(W_{0}^{1, p(.)}(\Omega)\right)^{\prime}$ is the dual space of $W_{0}^{1, p(.)}(\Omega)$ with respect to the inner product in $L^{2}(\Omega)$ and is defined as $W^{-1, p^{\prime}(.)}(\Omega)$, in the same way as the classical Sobolev spaces, where $\frac{1}{p(.)}+\frac{1}{p(.)^{\prime}}=1$.

- If $p \in C(\bar{\Omega}), q: \Omega \rightarrow[1,+\infty)$ is a measurable function and $\underset{x \in \Omega}{\operatorname{essinf}}\left(p^{*}(x)-q(x)\right)>0$ with $p^{*}(x)=\frac{n p(x)}{(n-p(x))_{2}}$, then $W_{0}^{1, p(.)}(\Omega) \hookrightarrow L^{q(.)}(\Omega)$ is continuous and compact.

Lemma 2.1. ([9]) Let $\Omega$ be a bounded domain of $\mathbb{R}^{n}, p($.$) and m($.$) satisfy (1.2) and$ (1.3), then

$$
B_{0}\|\nabla u\|_{m(.)} \geq\|u\|_{p(.)}, \text { for all } u \in W_{0}^{1, m(.)}(\Omega),
$$

where the optimal constant of Sobolev embedding $B_{0}$ is depend on $p_{1,2}$ and $|\Omega|$.

Lemma 2.2 (Poincaré's Inequality). ([9]) Let $\Omega$ be a bounded domain of $\mathbb{R}^{n}$ and $m($. satisfies (1.3), then

$$
D_{0}\|\nabla u\|_{m(.)} \geq\|u\|_{m(.)}, \text { for all } u \in W_{0}^{1, m(.)}(\Omega),
$$

where the optimal constant of Sobolev embedding $D_{0}$ is depend on $m_{1,2}$ and $|\Omega|$. 


\subsection{Mathematical assumptions}

In this section, we establish the blow-up result for solutions with positive energy. Let the function $f_{p(.)} \in C^{0}\left(\mathbb{R}, \mathbb{R}^{+}\right)$, with the primitive

$$
F(u)=\int_{0}^{u} f_{p(.)}(\eta) \mathrm{d} \eta
$$

satisfies

$$
\left|f_{p(.)}(s)\right| \leq C_{0}|s|^{p(.)-1}, \quad p(x) F(s) \leq s f_{p(.)}(s), s \in \mathbb{R}, C_{0}>0 .
$$

A simple typical example of these functions is

$$
f_{p(.)}(s)=|s|^{p(x)-2} s .
$$

Assume that $a(x, t)$ is a positive function which belongs to the space $W^{1, \infty}\left(0, \infty ; L^{\infty}(\Omega)\right)$ and that $a_{t}(x, t) \leq 0$ a.e. for $t \geq 0$. Let

$$
B_{1}=\max \left(1, B_{0},\left(\frac{1}{C_{0}}\right)^{\frac{1}{p_{1}}}\right), \quad \alpha_{1}=\left(\frac{1}{B_{1}^{p_{1}} C_{0}}\right)^{\frac{m_{2}}{p_{1}-m_{2}}}, \alpha_{0}=\left\|\nabla u_{0}\right\|_{m(.)}^{m_{2}},
$$

and

$$
E_{0}=\left(\frac{1}{B_{1}^{p_{1}} C_{0}}\right)^{\frac{m_{2}}{p_{1}-m_{2}}}\left(\frac{1}{m_{2}}-\frac{1}{p_{1}}\right)=\left(\frac{1}{m_{2}}-\frac{1}{p_{1}}\right) \alpha_{1} .
$$

\section{Main result}

In this section, we present our main blow-up result. We start with a local existence result for the problem (1.1), which can be established by combining the arguments of $[3,6]$, the following theorem, which confirms the existence of a local solution is a direct result.

Theorem 3.1. For all $u_{0} \in W_{0}^{1, m(.)}(\Omega)$, there exists a number $T_{0} \in(0, T]$ such that the problem (1.1) has a strong solution $u$ on $\left[0, T_{0}\right]$ satisfying

$$
u \in C\left(\left[0, T_{0}\right] ; W_{0}^{1, m(.)}(\Omega)\right) \cap C\left(\left[0, T_{0}\right] ; L^{p(.)}(\Omega)\right) \cap W^{1,2}\left(\left[0, T_{0}\right] ; L^{2}(\Omega)\right) .
$$

\section{Blow up for positive initial energy}

This section first presents our main blow-up result and its proof for the problem (1.1). For this purpose, we start by the following lemma defining the energy of the solution.

Lemma 4.1. The corresponding energy to problem (1.1) is given by

$$
E(t)=\int_{\Omega} \frac{1}{m(x)}|\nabla u(x, t)|^{m(x)} \mathrm{d} x-\int_{\Omega} F(u(x, t)) \mathrm{d} x,
$$

furthermore, by the easily verified formula

$$
\frac{\mathrm{d} E(t)}{\mathrm{d} t}=-\int_{\Omega} a(x, t) u_{t}^{2}(x, t) \mathrm{d} x \leq 0,
$$


the inequality $E(t) \leq E(0)$ is obtained.

Now, we are in a position to state our main theorem results.

Theorem 4.2. If the initial data $u_{0} \in W^{1, m(.)}(\Omega)$ are such that $u_{0} \neq 0$,

$$
E(0)=\int_{\Omega} \frac{1}{m(x)}\left|\nabla u_{0}(x)\right|^{m(x)} \mathrm{d} x-\int_{\Omega} F\left(u_{0}(x)\right) \mathrm{d} x \leq E_{0},
$$

then there exists $T^{*}$ such that $\limsup _{t \rightarrow T^{*}}\|u(., t)\|_{2}=+\infty$. Moreover, if $E(0)<E_{0}$, then the $T^{*}$ can be bounded above as:

$$
T^{*} \leq \frac{8\left\|\sqrt{a_{0}} u_{0}\right\|_{L^{2}(\Omega)}^{2}}{\left(p_{1}-2\right)^{2}\left(E_{0}-E(0)\right)},
$$

where $a(x, 0):=a_{0}$ and $u(x, 0):=u_{0}$.

In order to prove the main theorem, we recall the following lemmas.

Lemma 4.3. ([16, Lemma1.1] and [19, Logarithmic convexity methods]) Assume that $\varphi \in C^{2}([0, T))$ satisfying:

$$
\varphi^{\prime \prime} \varphi-(1+\alpha)\left(\varphi^{\prime}\right)^{2} \geq 0, \quad \alpha>0
$$

and

$$
\varphi(0)>0, \quad \varphi^{\prime}(0)>0
$$

then

$$
\varphi \rightarrow \infty \text { as } t \rightarrow t_{1} \leq t_{2}=\frac{\varphi(0)}{\alpha \varphi^{\prime}(0)} .
$$

Lemma 4.4. Suppose $E(0)<E_{0}$ and $\alpha_{1}<\alpha_{0} \leq B_{1}^{-m_{2}}$. Then it exists a constant $\alpha_{2}>\alpha_{1}$ such that:

$$
\|\nabla u\|_{m(.)}^{m_{2}} \geq \alpha_{2}>\alpha_{1} \text { for all } t \geq 0 .
$$

Proof. Thanks to (2.3) and (2.1), we have for any $t \geq 0$

$$
\begin{gathered}
E(t)=\int_{\Omega} \frac{1}{m(x)}|\nabla u(x, t)|^{m(x)} \mathrm{d} x-\int_{\Omega} F(u(x, t)) \mathrm{d} x \\
\geq \frac{1}{m_{2}} \min \left(\|\nabla u\|_{m(.)}^{m_{1}},\|\nabla u\|_{m(.)}^{m_{2}}\right)-\int_{\Omega} \frac{C_{0}}{p(x)}|u(x, t)|^{p(x)} \mathrm{d} x \\
\geq \frac{1}{m_{2}} \min \left(\|\nabla u\|_{m(.)}^{m_{1}},\|\nabla u\|_{m(.)}^{m_{2}}\right)-\frac{C_{0}}{p_{1}} \max \left(B_{1}^{p_{1}}\|\nabla u\|_{m(.)}^{p_{1}}, B_{1}^{p_{2}}\|\nabla u\|_{m(.)}^{p_{2}}\right) \\
=\frac{1}{m_{2}} \min \left(\alpha^{\frac{m_{1}}{m_{2}}}, \alpha\right)-\frac{C_{0}}{p_{1}} \max \left(\left(\alpha B_{1}^{m_{2}}\right)^{\frac{p_{1}}{m_{2}}},\left(\alpha B_{1}^{m_{2}}\right)^{\frac{p_{2}}{m_{2}}}\right):=g(\alpha), \forall \alpha \in[0,+\infty[
\end{gathered}
$$

where $\alpha=\|\nabla u\|_{m(.)}^{m_{2}}$. Now if we let

$$
h(\alpha)=\frac{1}{m_{2}} \alpha-\frac{C_{0}}{p_{1}}\left(\alpha B_{1}^{m_{2}}\right)^{\frac{p_{1}}{m_{2}}}
$$

Notice that $h(\alpha)=g(\alpha)$, for $0<\alpha<B_{1}^{-m_{2}}$. It is easy to check that the function $h(\alpha)$ is increasing for $0<\alpha<\alpha_{1}$ and decreasing for $\alpha_{1}<\alpha \leq+\infty$. 
Because $E(0)<E_{0}=h\left(\alpha_{1}\right)$, there exists a positive constant $\alpha_{2} \in\left(\alpha_{1},+\infty\right)$ such that $h\left(\alpha_{2}\right)=E(0)$. Then we have

$$
h\left(\alpha_{0}\right)=g\left(\alpha_{0}\right) \leq E(0)=h\left(\alpha_{2}\right) .
$$

It implies that $\alpha_{0} \geq \alpha_{2}>\alpha_{1}$.

To show that $\|\nabla u(x, t)\|_{m(.)}^{m_{2}} \geq \alpha_{2}$ we reason by absurd while supposing that

$$
\left\|\nabla u\left(x, t^{*}\right)\right\|_{m(.)}^{m_{2}}<\alpha_{2}
$$

for a some $t^{*}$. Then by the continuity of $\|\nabla u(., t)\|_{m(.)}$-norm with respect to time variable, one can choose $t^{*}$ such that

$$
\alpha_{2}>\left\|\nabla u\left(x, t^{*}\right)\right\|_{m(.)}^{m_{2}}>\alpha_{1}
$$

The monotonicity of $h(\alpha)$, gives

$$
E\left(t^{*}\right) \geq h\left(\|\nabla u(x, t)\|_{m(.)}^{m_{2}}\right)>h\left(\alpha_{2}\right)=E(0)
$$

it is impossible because $E(0) \geq E(t)$ for all $t \geq 0$. Then, for all time $t \geq 0$ :

$$
\|\nabla u\|_{m(.)}^{m_{2}} \geq \alpha_{2}>\alpha_{1} \text {. }
$$

Proof of Theorem 1. Case 1: $E(0)<E_{0}$. The goal is to construct a suitable function which satisfies the conditions in Lemma (4.3). Following the arguments of [22, 23], for our purpose, we define the following suitable function

$$
\begin{gathered}
\varphi(t)=\int_{0}^{t} \int_{\Omega} a(x, s) u^{2}(x, s) \mathrm{d} x \mathrm{~d} s+\int_{0}^{t} \int_{\Omega}(s-t) a_{t}(x, s) u^{2}(x, s) \mathrm{d} x \mathrm{~d} s \\
+\left(T_{0}-t\right) \int_{\Omega} a_{0}(x) u_{0}^{2}(x) \mathrm{d} x+\beta\left(t+t_{0}\right)^{2}, t<T_{0}
\end{gathered}
$$

where $t_{0}, T_{0}$ and $\beta$ are positive constants to be determined later. Then using equation (1.1) and integration by parts, to obtains

$$
\begin{gathered}
\varphi^{\prime}(t)=\int_{\Omega} a(x, t) u^{2}(x, t) \mathrm{d} x-\int_{0}^{t} \int_{\Omega} a_{t}(x, s) u^{2}(x, s) \mathrm{d} x \mathrm{~d} s \\
-\int_{\Omega} a_{0}(x) u_{0}^{2}(x) \mathrm{d} x+2 \beta\left(t+t_{0}\right) \\
=2 \int_{0}^{t} \int_{\Omega} a(x, s) u(x, s) u_{t}(x, s) \mathrm{d} x \mathrm{~d} s+2 \beta\left(t+t_{0}\right),
\end{gathered}
$$

and

$$
\varphi^{\prime \prime}(t)=2 \int_{\Omega} a(x, t) u(x, t) u_{t}(x, t) \mathrm{d} x+2 \beta .
$$


Then, due to (2.4) and (4.6), the following is obtained

$$
\begin{aligned}
& \varphi^{\prime \prime}(t) \geq-2 \int_{\Omega}|\nabla u(x, t)|^{m(x)} \mathrm{d} x+2 \int_{\Omega} p(x) F(u) \mathrm{d} x+2 \beta \\
& \geq-2 \int_{\Omega}|\nabla u(x, t)|^{m(x)} \mathrm{d} x+2 p_{1}\left(\int_{\Omega} \frac{1}{m(x)}|\nabla u(x, t)|^{m(x)} \mathrm{d} x-E(t)\right)+2 \beta \\
& \geq\left(\frac{2 p_{1}}{m_{2}}-2\right) \int_{\Omega}|\nabla u(x, t)|^{m(x)} \mathrm{d} x-2 p_{1} E(t)+2 \beta \\
& \geq\left(\frac{2 p_{1}}{m_{2}}-2\right) \int_{\Omega}|\nabla u(x, t)|^{m(x)} \mathrm{d} x \\
& +2 p_{1} \int_{0}^{t} \int_{\Omega} a(x, s) u_{t}^{2}(x, s) \mathrm{d} x \mathrm{~d} s-2 p_{1} E(0)+2 \beta \\
& \geq\left(\frac{2 p_{1}}{m_{2}}-2\right) \min \left(\|\nabla u\|_{m(.)}^{m_{1}},\|\nabla u\|_{m(.)}^{m_{2}}\right) \\
& +2 p_{1} \int_{0}^{t} \int_{\Omega} a(x, s) u_{t}^{2}(x, s) \mathrm{d} x \mathrm{~d} s-2 p_{1} E(0)+2 \beta \\
& \geq\left(\frac{2 p_{1}}{m_{2}}-2\right) \min \left(\alpha_{2}^{\frac{m_{1}}{m_{2}}}, \alpha_{2}\right) \\
& +2 p_{1} \int_{0}^{t} \int_{\Omega} a(x, s) u_{t}^{2}(x, s) \mathrm{d} x \mathrm{~d} s-2 p_{1} E(0)+2 \beta \\
& \geq 2 p_{1}\left(\frac{1}{m_{2}}-\frac{1}{p_{1}}\right) \min \left(\alpha_{1}^{\frac{m_{1}}{m_{2}}}, \alpha_{1}\right) \\
& -2 p_{1} E(0)+2 \beta+2 p_{1} \int_{0}^{t} \int_{\Omega} a(x, s) u_{t}^{2}(x, s) \mathrm{d} x \mathrm{~d} s \\
& =2 p_{1}\left(\frac{1}{m_{2}}-\frac{1}{p_{1}}\right) \alpha_{1}-2 p_{1} E(0) \quad(\text { by }(2.5)) \\
& +2 \beta+2 p_{1} \int_{0}^{t} \int_{\Omega} a(x, s) u_{t}^{2}(x, s) \mathrm{d} x \mathrm{~d} s \\
& =2 p_{1}\left(E_{0}-E(0)\right)+2 \beta+2 p_{1} \int_{0}^{t} \int_{\Omega} a(x, s) u_{t}^{2}(x, s) \mathrm{d} x \mathrm{~d} s
\end{aligned}
$$

Now, let $\beta=2\left(E_{0}-E(0)\right)>0$, and note that $p_{1}>2$, then

$$
\varphi^{\prime \prime}(t) \geq\left(p_{1}+2\right) \beta+\left(p_{1}+2\right) \int_{0}^{t} \int_{\Omega} a(x, s) u_{t}^{2}(x, s) \mathrm{d} x \mathrm{~d} s
$$

From (4.7), (4.8), (4.9) and (4.10), we have

$$
\left\{\begin{array}{c}
\varphi(0)=T_{0} \int_{\Omega} a_{0}(x) u_{0}^{2}(x) \mathrm{d} x+\beta t_{0}^{2}>0 ; \\
\varphi^{\prime}(0)=2 \beta t_{0}>0 \\
\varphi^{\prime \prime}(t) \geq\left(p_{1}+2\right) \beta>0 \forall t \geq 0 .
\end{array}\right.
$$


Therefore $\varphi$ and $\varphi^{\prime}$ are both positive. Since $a_{t}(x, t) \leq 0$, for all $x \in \Omega$ and $t \geq 0$, we have

$$
\varphi(t) \geq \int_{0}^{t} \int_{\Omega} a(x, s) u^{2}(x, s) \mathrm{d} x \mathrm{~d} s+\beta\left(t+t_{0}\right)^{2},
$$

Thus, from (4.7)-(4.10) and (4.11), the following inferred for all $(\zeta, \eta) \in \mathbb{R}^{2}$

$$
\begin{gathered}
\varphi(t) \zeta^{2}+\varphi^{\prime}(t) \zeta \eta+\frac{\eta^{2}}{p_{1}+2} \varphi^{\prime \prime}(t) \\
\geq\left(\int_{0}^{t} \int_{\Omega} a(x, s) u^{2}(x, s) \mathrm{d} x \mathrm{~d} s+\beta\left(t+t_{0}\right)^{2}\right) \zeta^{2} \\
+2 \zeta \eta \int_{0}^{t} \int_{\Omega} a(x, s) u(x, s) u_{t}(x, s) \mathrm{d} x \mathrm{~d} s+2 \zeta \eta \beta\left(t+t_{0}\right) \\
+\beta \eta^{2}+\eta^{2} \int_{0}^{t} \int_{\Omega} a(x, s) u_{t}^{2}(x, s) \mathrm{d} x \mathrm{~d} s \geq 0,
\end{gathered}
$$

which implies that

$$
\varphi(t) \frac{\varphi^{\prime \prime}(t)}{p_{1}+2}-\left(\frac{\varphi^{\prime}(t)}{2}\right)^{2} \geq 0
$$

subsequently

$$
\varphi(t) \varphi^{\prime \prime}(t)-\frac{p_{1}+2}{4}\left(\varphi^{\prime}(t)\right)^{2} \geq 0 .
$$

Then using Lemma (4.3), to infer $\varphi(t) \rightarrow \infty$ as $t \rightarrow T^{*}$, where,

$$
T^{*} \leq \frac{\varphi(0)}{\left(\frac{p_{1}-2}{4}\right) \varphi^{\prime}(0)}=\frac{2\left(T_{0}\left\|\sqrt{a_{0}} u_{0}\right\|_{L^{2}(\Omega)}^{2}+\beta t_{0}^{2}\right)}{\left(p_{1}-2\right) \beta t_{0}} .
$$

Now we go to choose appropriate $t_{0}$ and $T_{0}$. Let $t_{0}$ be any number which depends only on $p_{1}, E_{0}-E(0)$ and $\left\|u_{0}\right\|_{L^{2}(\Omega)}$ as

$$
t_{0}>\frac{\left\|\sqrt{a_{0}} u_{0}\right\|_{L^{2}(\Omega)}^{2}}{\left(p_{1}-2\right)\left(E_{0}-E(0)\right)} .
$$

Fix $t_{0}$, then $T_{0}$ can be picking as

$$
T_{0}=\frac{2\left(T_{0}\left\|\sqrt{a_{0}} u_{0}\right\|_{L^{2}(\Omega)}^{2}+\beta t_{0}^{2}\right)}{\left(p_{1}-2\right) \beta t_{0}},
$$

so that

$$
T_{0}=\frac{2\left(E_{0}-E(0)\right) t_{0}^{2}}{\left(p_{1}-2\right)\left(E_{0}-E(0)\right) t_{0}-\left\|\sqrt{a_{0}} u_{0}\right\|_{L^{2}(\Omega)}^{2}},
$$


Therefore the lifespan of the solution $u(x, t)$ is bounded by

$$
\begin{gathered}
T^{*} \leq \inf _{t \geq t_{0}} \frac{2\left(E_{0}-E(0)\right) t^{2}}{\left(p_{1}-2\right)\left(E_{0}-E(0)\right) t-\left\|\sqrt{a_{0}} u_{0}\right\|_{L^{2}(\Omega)}^{2}}, \\
=\frac{8\left\|\sqrt{a_{0}} u_{0}\right\|_{L^{2}(\Omega)}^{2}}{\left(p_{1}-2\right)^{2}\left(E_{0}-E(0)\right)} .
\end{gathered}
$$

Case 2: $E(0)=E_{0}$. For this case, actually we consider the following claim

Claim 4.5. There exists $t^{*}>0$ such that $E\left(t^{*}\right)<E_{0}$.

Suppose Claim is not true which means that $E(t)=E_{0}$ for all $t \geq 0$. Then by the continuity of $\|\nabla u(., t)\|_{m(.)}$ there exists a $t_{0}$ small enough, such that

$$
E(t)=E_{0} \text { and }\|\nabla u(., t)\|_{m(.)}^{m_{2}} \geq \alpha_{2}>\alpha_{1} \text { for all } t \in\left[0, t_{0}\right]
$$

Then we consider the solution of $(1.1)$ on $\left[0, t_{0}\right]$,

$$
0=E(t)-E_{0}=-\int_{0}^{t_{0}} \int_{\Omega} a(x, t) u_{t}^{2}(x, t) \mathrm{d} x \mathrm{~d} t
$$

which turns out to be

$$
\int_{\Omega} a(x, t) u_{t}(x, t) u(x, t) \mathrm{d} x=0 \text { a.e. on }\left[0, t_{0}\right]
$$

And consequently, due to the equation (1.1),

$$
\begin{gathered}
\int_{\Omega} a(x, t) u_{t}(x, t) u(x, t) \mathrm{d} x \\
=-\int_{\Omega}|\nabla u(x, t)|^{m(x)} \mathrm{d} x+\int_{\Omega} u(x, t) f_{p(.)}(u(x, t)) \mathrm{d} x=0 \text { a.e.on }\left(0, t_{0}\right] .
\end{gathered}
$$

On the other hand,

$$
\begin{gathered}
E_{0}=E(t)=\int_{\Omega} \frac{1}{m(x)}|\nabla u(x, t)|^{m(x)} \mathrm{d} x-\int_{\Omega} F(u(x, t)) \mathrm{d} x \\
\geq \frac{1}{m_{2}} \int_{\Omega}|\nabla u(x, t)|^{m(x)} \mathrm{d} x-\frac{1}{p_{1}} \int_{\Omega} u(x, t) f_{p(.)}(u(x, t)) \mathrm{d} x \\
=\left(\frac{1}{m_{2}}-\frac{1}{p_{1}}\right) \int_{\Omega}|\nabla u(x, t)|^{m(x)} \mathrm{d} x(\text { by }(4.13)) \\
>\left(\frac{1}{m_{2}}-\frac{1}{p_{1}}\right) \min \left(\alpha_{1}^{\frac{m_{1}}{m_{2}}}, \alpha_{1}\right)(\text { by }(4.6)) \\
=\left(\frac{1}{m_{2}}-\frac{1}{p_{1}}\right) \alpha_{1}=E_{0}(\text { by }(2.5) \text { and }(2.6))
\end{gathered}
$$

which is a contradiction.

The proof of Theorem (4.2) is complete since one can apply the previous case (Case 1) after changing the time origin to $t^{*}$. 


\section{Blow up for negative initial energy}

This section is devoted to the main blow-up result and its proof in the case when $E(0) \leq 0$.

Assume that $a(x, t)$ is a positive function which belongs to the space $W^{1, \infty}\left(0, \infty ; L^{\infty}(\Omega)\right)$ and that $a_{t}(x, t) \geq 0$ a.e. for $t \geq 0$.

The next Lemma gives the desired blow-up result.

Lemma 5.1. Let $u_{0} \in W_{0}^{1, m(.)}(\Omega)$ such that $\int_{\Omega} u_{0}^{2} \mathrm{~d} x>0, f_{p(.)}$ satisfies (2.4) and $E(0) \leq 0$. Then there exists a finite time $T_{\max }<\infty$ such that

$$
\int_{\Omega}|u(t)|^{2} \mathrm{~d} x \rightarrow \infty \text { if } t \rightarrow T_{\max } .
$$

Proof of Lemma (5.1). We then define

$$
\phi(t)=\frac{1}{2} \int_{\Omega} a(x, t)|u(t)|^{2} \mathrm{~d} x
$$

Differentiating $\phi$ with respect to $t$, gets

$$
\begin{gathered}
\phi^{\prime}(t)=\int_{\Omega} a(x, t) u u_{t} \mathrm{~d} x+\frac{1}{2} \int_{\Omega} a_{t}(x, t)|u(t)|^{2} \mathrm{~d} x \\
\geq-\int_{\Omega}\left(|\nabla u|^{m(x)}-u f_{p(.)}(u)\right) \mathrm{d} x \quad(\text { by }(1.1)) \\
\geq-\int_{\Omega}\left(|\nabla u|^{m(x)}-p(x) F(u)\right) \mathrm{d} x \quad(\text { by }(2.4)) \\
\geq-\int_{\Omega}|\nabla u|^{m(x)} \mathrm{d} x+p_{1} \int_{\Omega} F(u) \mathrm{d} x \\
=-\int_{\Omega}|\nabla u|^{m(x)} \mathrm{d} x+p_{1} \int_{\Omega} \frac{1}{m(x)}|\nabla u(x, t)|^{m(x)} \mathrm{d} x-p_{1} E(t) \quad(\text { by }(4.1)) \\
\geq\left(\frac{p_{1}}{m_{2}}-1\right) \int_{\Omega}|\nabla u|^{m(x)} \mathrm{d} x-p_{1} E(0) \quad(\text { by }(4.2)) \\
\geq\left(\frac{p_{1}}{m_{2}}-1\right) \int_{\Omega}|\nabla u|^{m(x)} \mathrm{d} x=c_{0} \int_{\Omega}|\nabla u|^{m(x)} \mathrm{d} x, \quad\left(c_{0}>0\right)
\end{gathered}
$$

We define the sets

$$
\Omega_{2}=\{x \in \Omega|| \nabla u \mid \geq 1\} \text { and } \Omega_{1}=\{x \in \Omega|| \nabla u \mid<1\} .
$$

So

$$
\begin{gathered}
\phi^{\prime}(t) \geq c_{0} \int_{\Omega_{2}}|\nabla u|^{m_{1}} \mathrm{~d} x+c_{0} \int_{\Omega_{1}}|\nabla u|^{m_{2}} \mathrm{~d} x \\
\geq C_{1}\left(\left(\int_{\Omega_{2}}|\nabla u|^{2} \mathrm{~d} x\right)^{\frac{m_{1}}{2}}+\left(\int_{\Omega_{1}}|\nabla u|^{2} \mathrm{~d} x\right)^{\frac{m_{2}}{2}}\right),
\end{gathered}
$$


Using the fact that $\|\nabla u\|_{2} \leq C\|\nabla u\|_{q}$, for all $q \geq 2$, to obtain

$$
\left\{\begin{array}{l}
\left(\phi^{\prime}(t)\right)^{\frac{2}{m_{2}}} \geq C_{2} \int_{\Omega_{1}}|\nabla u|^{2} \mathrm{~d} x ; \\
\left(\phi^{\prime}(t)\right)^{\frac{2}{m_{1}}} \geq C_{3} \int_{\Omega_{2}}|\nabla u|^{2} \mathrm{~d} x .
\end{array}\right.
$$

By addition, leads to

$$
\begin{aligned}
& \left(\phi^{\prime}(t)\right)^{\frac{2}{m_{2}}}+\left(\phi^{\prime}(t)\right)^{\frac{2}{m_{1}}} \geq C_{4} \int_{\Omega}|\nabla u|^{2} \mathrm{~d} x \\
\geq & C_{5} \int_{\Omega}|u|^{2} \mathrm{~d} x \geq \frac{C_{5}}{\sup a(x, t)} \phi(t), \forall t \geq 0 .
\end{aligned}
$$

or

$$
\left(\phi^{\prime}(t)\right)^{\frac{2}{m_{1}}}\left(1+\left(\phi^{\prime}(t)\right)^{\frac{2}{m_{2}}-\frac{2}{m_{1}}}\right) \geq C_{6} \phi(t), \forall t \geq 0 .
$$

By (5.1) and the fact that $\phi(t) \geq \phi(0)>0\left(\phi^{\prime}(t) \geq 0\right)$, we have, for each $t>0$, either

$$
\left\{\begin{array}{c}
\left(\phi^{\prime}(t)\right)^{\frac{2}{m_{1}}} \geq \frac{C_{6}}{2} \phi(t) \geq \frac{C_{6}}{2} \phi(0) \\
\text { or }\left(\phi^{\prime}(t)\right)^{\frac{2}{m_{2}}} \geq \frac{C_{6}}{2} \phi(t) \geq \frac{C_{6}}{2} \phi(0)
\end{array}\right.
$$

which gives, in turn

$$
\left\{\begin{array}{c}
\phi^{\prime}(t) \geq C_{7}(\phi(0))^{\frac{m_{2}}{2}} \\
\text { or } \phi^{\prime}(t) \geq C_{8}(\phi(0))^{\frac{m_{1}}{2}}
\end{array}\right.
$$

hence

$$
\phi^{\prime}(t) \geq \alpha=\min \left(C_{7}(\phi(0))^{\frac{m_{2}}{2}}, C_{8}(\phi(0))^{\frac{m_{1}}{2}}\right),
$$

since $\frac{1}{p_{2}}-\frac{1}{p_{1}} \leq 0,(5.2)$ yields

$$
\left(\phi^{\prime}(t)\right)^{\frac{2}{m_{1}}}(1+\alpha)^{\frac{2}{m_{2}}-\frac{2}{m_{1}}} \geq C_{4} \phi(t), \forall t \geq 0 .
$$

therefore

$$
\phi^{\prime}(t) \geq \beta \phi^{\frac{m_{1}}{2}}(t), \forall t \geq 0 .
$$

simple integrating then leads to

$$
(\phi(t))^{1-\frac{m_{1}}{2}} \leq(\phi(0))^{1-\frac{m_{1}}{2}}-\frac{m_{1}-2}{2} \beta t, \forall t \geq 0 .
$$

which implies that

$$
\phi(t) \geq \frac{1}{\left((\phi(0))^{1-\frac{m_{1}}{2}}-\frac{m_{1}-2}{2} \beta t\right)^{\frac{2}{m_{1}-2}}}
$$

This show that $\phi$ blows up in finite time $T_{\max }$ given by the estimate

$$
T_{\max } \leq \frac{2(\phi(0))^{1-\frac{m_{1}}{2}}}{\left(m_{1}-2\right) \beta} .
$$

Acknowledgements. The authors would like to thank the anonymous referees and the handling editor for their reading and for relevant remarks/suggestions. 


\section{References}

[1] Abita, R., Blow-up phenomenon for a semilinear pseudo-parabolic equation involving variable source, Applicable Analysis, 2021.

[2] Abita, R., Bounds for below-up time in a nonlinear generalized heat equation, Applicable Analysis, 2020.

[3] Abita, R., Benyattou, B., Quasilinear parabolic equations with $p(x)$-laplacian diusion terms and nonlocal boundary conditions, Stud. Univ. Babeş-Bolyai Math., 64(2019), 101-116.

[4] Acerbi, E., Mingione, G., Regularity results for stationary eletrorheological fluids, Arch. Ration. Mech. Anal, 164(2002), 213-259.

[5] Aiguo, B., Xianfa, S., Bounds for the blowup time of the solutions to quasi-linear parabolic problems, Zeitschrift für Angewandte Mathematik und Physik (ZAMP), 65(2014).

[6] Akagi, G., Ôtani, M., Evolutions inclusions governed by subdifferentials in reflexive Banach spaces, J. Evol. Equ., 4(2004), 519-541.

[7] Antonsev, S.N., Blow up of solutions to parabolic equations with nonstandard growth conditions, J. Comput. Appl. Math., 234(2010), 2633-2645.

[8] Baghaei, K., Ghaemi, M.B., Hesaaraki, M., Lower bounds for the blow-up time in a semilinear parabolic problem involving a variable source, Applied Mathematics Letters, 27(2014), 49-52.

[9] Diening, L., Hästo, P., Harjulehto, P., Ruzicka, M., Lebesgue and Sobolev Spaces with Variable Exponents, in: Springer Lecture Notes, Springer-Verlag, Berlin, 2011 and 2017.

[10] Diening, L., Ruzicka, M., Calderon Zygmund operators on generalized Lebesgue spaces $L^{p(x)}(\Omega)$ and problems related to fluid dynamics, Preprint Mathematische Fakultät, Albert-Ludwigs-Universität Freiburg, Freiburg, 120((21/2002,04.07.2002)), 197-220.

[11] Fan, X., Shen, J., Zhao, D., Sobolev embedding theorems for spaces $W^{k, p(x)}(\Omega)$, J. Math. Anal. Appl., 262(2001), 749-760.

[12] Ferreira, R., de Pablo, A., Pérez-Llanos, M., Rossi, J.D., Critical exponents for a semilinear parabolic equation with variable reaction, Proceedings of the Royal Society of Edinburgh Section A Mathematics, 142A(2012), 1027-1042.

[13] Fu, Y., The existence of solutions for elliptic systems with nonuniform growth, Studia Math., 151(2002), 227-246.

[14] Fujita, H., On the blowing up of solutions of the Cauchy problem for $u_{t}=\Delta u+u^{1+\alpha}$, J. Fac. Sci. Univ. Tokyo Sect., 13(1966), no. I, 109-124.

[15] Hua, W., Yijun, H., On blow-up of solutions for a semilinear parabolic equation involving variable source and positive initial energy, Applied Mathematics Letters, 26(2013), no. 10, 1008-1012.

[16] Kalantarov, V., Ladyzhenskaya, O.A., The occurence of collapse for quasilinear equation of paprabolic and hyperbolic types, J. Sov. Math., 10(1978), 53-70.

[17] Kovàcik, O., Rákosnik, J., On spaces $L^{p(x)}(\Omega)$ and $W^{1, p(x)}(\Omega)$, Czechoslovak Math. J., 41(1991).

[18] Ni, W.M., Sacks, P.E., Tavantzis, J., On the asymptotic behavior of solutions of certain quasilinear parabolic equations, J. Differential Equations, 54(1984), 97-120.

[19] Payne, L.E., Improperly Posed Problems in Partial Differential Equations, Regional Conference Series in Applied Mathematics, 1975, 1-61. 
[20] Xiulan, W., Guo, B., Wenjie, G., Blow-up of solutions for a semilinear parabolic equation involving variable source and positive initial energy, Applied Mathematics Letters, 26(2013), 539-543.

[21] Zhong, T., The reaction-diffusion equation with lewis function and critical sobolev exponent, Journal of Mathematical Analysis and Applications, 272(2002), no. 2, 480-495.

[22] Zhou, Y., Global nonexistence for a quasilinear evolution equation with critical lower energy, Arch. Inequal. Appl., 2(2004), 41-47.

[23] Zhou, Y., Global nonexistence for a quasilinear evolution equation with a generalized lewis function, Journal for Analysis and its Applications, 24(2005), 179-187.

\author{
Abita Rahmoune \\ Department of Technical Sciences, \\ 03000 Laghouat University, Algeria \\ e-mail: abitarahmoune@yahoo.fr \\ Benyattou Benabderrahmane \\ e-mail: benyattou.benabderrahmane@univ-msila.dz \\ Laboratory of Pure and Applied Mathematics, \\ Mohamed Boudiaf University-M'Sila 28000, Algeria
}

